\title{
Increase in HIV-1-transmitted drug resistance among ART-naïve youths at the China-Myanmar border during 2009 2017
}

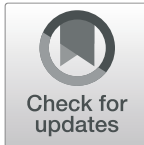

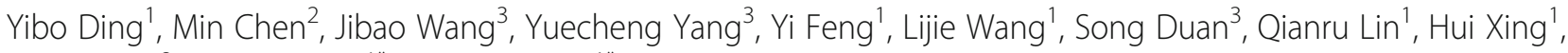
Yanling $\mathrm{Ma}^{2}$, Mengjie $\mathrm{Han}^{1 *}$ and Liying $\mathrm{Ma}^{1 *}$

\begin{abstract}
Background: HIV-transmitted drug resistance (TDR) is found in antiretroviral therapy (ART)-naïve populations infected with HIV-1 with TDR mutations and is important for guiding future first- and second-line ART regimens. We investigated TDR and its effect on CD4 count in ART-naïve youths from the China-Myanmar border near the Golden Triangle to better understand TDR and effectively guide ART.

Methods: From 2009 to 2017, 10,832 HIV-1 infected individuals were newly reported along the Dehong border of China, 573 ART-naïve youths (16 25 y) were enrolled. CD4 counts were obtained from whole blood samples. HIV pol gene sequences were amplified from RNA extracted from plasma. The Stanford REGA program and jpHMM recombination prediction tool were used to determine genotypes. TDR mutations (TDRMs) were analyzed using the Stanford Calibrated Population Resistance tool.

Results: The most common infection route was heterosexuals (70.51\%), followed by people who inject drugs (PWID, 19.20\%) and men who have sex with men (MSM) (8.90\%). The distribution of HIV genotypes mainly included the unique recombinant form (URF) (44.08\%), 38.68\% were CRFs, $13.24 \%$ were subtype $C$ and $4.04 \%$ were subtype B. The prevalence of TDR increased significantly from 2009 to 2017 (3.48 to 9.48\%) in ART-naïve youths (4.00 to 13.16\% in Burmese subjects, 3.33 to 5.93\% in Chinese subjects), and the resistance to non-nucleoside reverse transcriptase inhibitors (NNRTIs), nucleoside and nucleotide reverse transcriptase inhibitors (NRTIs), and protease inhibitors (PIs) were 3.49, 2.62, and 0.52\%, respectively. Most $(94.40 \%, n=34)$ of HIV-1-infected patients with TDRM had mutation that conferred resistance to a single drug class. The most common mutations $\mathrm{Y} 181 \mathrm{I} / \mathrm{C}$ and K103N, were found in 7 and 9 youths, respectively. The mean CD4 count was significantly lower among individuals with

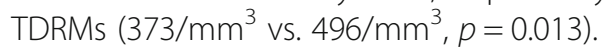

Conclusions: The increase in the prevalence of HIV-1 TDR increase and a low CD4 count of patients with TDRMs in the China-Myanmar border suggests the need for considering drug resistance before initiating ART in HIV recombination hotspots.
\end{abstract}

Keywords: HIV, Transmitted drug resistance, CD4 count, Hotspots

\footnotetext{
*Correspondence: mjhan@chinaaids.cn; mal@chinaaids.cn

${ }^{1}$ State Key Laboratory of Infectious Disease Prevention and Control, National Center for AIDS/STD Control and Prevention, Collaborative Innovation Center for Diagnosis and Treatment of Infectious Diseases, Chinese Center for Disease Control and Prevention, 155 Changbai Road, Changping District, Beijing 102206, China

Full list of author information is available at the end of the article
}

(c) The Author(s). 2021 Open Access This article is licensed under a Creative Commons Attribution 4.0 International License, which permits use, sharing, adaptation, distribution and reproduction in any medium or format, as long as you give appropriate credit to the original author(s) and the source, provide a link to the Creative Commons licence, and indicate if changes were made. The images or other third party material in this article are included in the article's Creative Commons licence, unless indicated otherwise in a credit line to the material. If material is not included in the article's Creative Commons licence and your intended use is not permitted by statutory regulation or exceeds the permitted use, you will need to obtain permission directly from the copyright holder. To view a copy of this licence, visit http://creativecommons.org/licenses/by/4.0/ The Creative Commons Public Domain Dedication waiver (http://creativecommons.org/publicdomain/zero/1.0/) applies to the data made available in this article, unless otherwise stated in a credit line to the data. 


\section{Background}

A total of 37.9 million people living with HIV (PLWH) worldwide, and 24.5 million people were receiving antiretroviral therapy (ART) at the end of 2018 [1]. However, the problem of drug resistance (DR) has been a focus with the increasing use of ART. DR, including transmitted drug resistance (TDR) and acquired drug resistance (ADR), develops because of viral replication in the presence of ART drugs. TDR is found in ART-naïve populations infected with the virus carrying DR mutations. TDR surveys effectively guide future first- and second-line ART regimens [2].

The monitoring of population levels of DR is also critical to achieving the WHO/UNAIDS 90-90-90 targets. The WHO HIV drug resistance 2019 report noted that 12 of the 18 countries that reported survey findings of TDR had reached levels above $10 \%$. The 2019 report also reported that the average TDR of the three countries bordering China exceeded the 5\% moderate level: Myanmar 5.40\% (3.10\% 9.20\%), Vietnam 5.80\% (3.40\% $\sim 9.50 \%)$, and Nepal $12.90 \%$ (8.80\% 18.50\%). Overall, the average TDR among newly reported HIV individuals is relatively low $(4.10 \%)$ in most recently research in China [3]. Another national survey in 2015 reported that [4], the average TDR of Chinese youth was 3.6\% (32/ 894 ), including $3.00 \%$ of MSM and $5.80 \%$ of heterosexual transmission. However, estimates of the rates of TDR in the HIV epidemic vary throughout China. Several surveys reported that the TDR of some cities in Yunnan Province of China exceeded the WHO 5\% moderate prevalence level $[5,6]$.

Yunnan Province is in the southwestern part of China, bordering Myanmar, Vietnam, and Laos. By the end of 2016, the number of PLWH in Yunnan $(91,986)$ was the second-highest of all provinces in China; of these, 70,577 (76.7\%) were receiving ART. Dehong city is a major city for trading in the Yunnan-Myanmar border area. Dehong shares an international border with Kachin and the Shan state, Myanmar, which are two of the major states of the "Golden Triangle". The "Golden Triangle" is one of the world's largest drug production centers [7], and there is a severe HIV transmission problem around this area. The first HIV spread in Chinese people who inject drugs (PWID) was found in Dehong [8].

The prevalent HIV-1 stains (92.3\%) in most regions of China are CRF01_AE, CRF07_BC, subtype B'/B, CRF08_ BC. Subtype C, URFs or other CRFs were less than $3 \%$ [9]. However, Dehong City is a hotspot of HIV recombination and transmission, [10] from which most of the HIV-1 strains currently circulating in China first appeared [11-14]. In a previous survey of HIV-1-infected youths in Yunnan found that the URF were as high as $64 \%$ [15]. Whether frequent recombination increases the spread of drug resistance in treatment-naïve population is not clear. It is essential to monitor TDR in this recombination and transmitted hotspots.

According to the WHO HIV drug resistance (HIVDR) threshold survey method [16], untreated youths $(<25 \mathrm{y})$ are more likely to have recent infection and are representative of TDR $[17,18]$. To better understand TDR and its effect on CD4 count, we investigated TDRMs and HIV genotypes in youths over a 9-year period and examined the effect of TDRMs on CD4 counts in the China-Myanmar border near the "Golden Triangle".

\section{Methods}

\section{Study population and ethical review}

The ethical review of this study was formally approved by and obtained written consent (approval no. X190111549) from the Medical Ethics Certification Committee of the Chinese Center for Disease Control and Prevention. Written informed consent was obtained from all subjects included in the study.

From 2009 to 2017, a total of 10,832 people were newly reported with HIV-1 infection at the Dehong border of China. Among these individuals, 2210 were youths $(<25$ y). We amplified the HIV-1 pol gene from the plasma of 666 of these youths who were chosen according to the following criteria: 1) $16 \sim 25 \mathrm{y}$, newly diagnosed with HIV within 3-6 months, and not motherto-infant transmission; 2) never received ART; 3) agreed to provide written informed consent and to allow plasma samples to be collected and stored for follow-up studies; and 4) agreed to provide epidemiological information. Finally, $573(86 \%, 573 / 666)$ youths from whom the viral pol gene was successfully amplified were used in the analysis. The annual sampling percentage for this study, were $13.58 \%$ in $2009 \sim 2011,22.65 \%$ in $2012 \sim 2013$, $26.90 \%$ in $2014 \sim 2015$, and $52.25 \%$ in $2016 \sim 2017$ (supplemental Table S1).

\section{CD4 count and pol gene amplification}

CD4+ T lymphocyte were counted using FACSCalibur flow cytometer and supporting kits (BD Bioscience, United States). The CD4 counts of $461(80 \%)$ of the youths in the study were recorded. RNA was extracted from $140 \mu \mathrm{L}$ of plasma samples using the QIAamp RNA Blood Mini Kit (Qiagen, Hilden, Germany). The partial pol gene, including the protease and reverse transcriptase (PR and RT) coding regions, was amplified by nested polymerase chain reaction (PCR) and an "in-house" method [19] by one-step RT-PCR, using the primers F1a (TGAARGAITGYACTGARAGRCAGGCTAAT), F1b (ACTGARAGRCAGGCTAATTTTTTAG), and RT-R1 (ATCCCTGCATAAATCTGACTTGC). The primers used in nested PCR were PRT-F2 (CTTTARCTTC CCTCARATCACTCT) and RT-R2 (CTTCTGTATG TCATTGACAGTCC). PR/RT covered a fragment of 
1056 bps corresponding to nucleotides 2259 to 3314 relative to the HXB2 genome. The positive PCR amplicons were purified using the QIAquick Gel Extraction Kit (Qiagen, Valencia, CA) and sequenced. Amplicons were purified using the Illustra GFXR PCR DNA and Gel Band Purification Kit (GE Healthcare, United Kingdom) according to the manufacturer's recommendations. The purified DNA was sequenced using the Big Dye Terminator Cycle Sequencing Ready Reaction Kit v.3.1 (Applied Biosystems, CA, United States) with processing on an automated ABI 3130xl sequencer (Applied Biosystems) using Sanger's method. All sequence data were spliced and cleaned using Sequencher v5.1 (Gene Codes Corporation).

\section{Genotype identification and analysis}

Sequences were edited in AliView software and aligning using the Los Alamos HIV Align tool with HXB2 reference sequences. The phylogenetic tree was approximated using the maximum likelihood method with general time-reversible (GTR) modeling with RAxML (version 8) software [20] and Figtree v1.4.3. Identification of HIV-1 genotypes was perform using the phylogenetic tree and the Stanford REGA HIV-1 Subtyping Tool 3.0 [21]. If the results of the REGA tool were 'Recombination', 'Recombination-like', 'potential-Recombination', 'check the report', the phylogenetic tree was used for confirmation.

\section{Drug resistance mutations and analysis}

TDRMs were defined as the proportion of surveillance drug resistance mutations (SDRMs), identified by the Stanford Calibrated Population Resistance (CPR) tool 8.0 (last updated on 2019-07-01), according to the WHO2009 SDRM list [22]. The Stanford HIVDB Program 8.9.1 (last updated on 2019-10-25, https://hivdb.stanford. $\mathrm{edu} / \mathrm{hivdb} /$ ) was used to score resistance to protease inhibitors (PIs), nucleoside reverse transcriptase inhibitors (NRTIs) and nonnucleoside reverse transcriptase inhibitors (NNRTIs) [23]. Sequences were determined to be susceptible $(<15$, including potential resistance) or resistant $(15 \leq$ low $<30,30 \leq$ medium $<60$, or high $>60)$ based on their scores.

\section{Statistical analysis}

Statistical analyses were perform using R 3.6.2 and RStudio 1.1.463. The Chi-square test or Fisher's exact test was used to verify differences in the distribution of demographic and clinical characteristics and genotypes and report proportions of TDRMs or network inclusion by participant characteristics (for example, nationality, educational status, etc.). The Cochran-Armitage trend test was used to test trends such as TDR trend and the proportion of genotypes changed. When appropriate, $p<0.05$ was defined as statistically significant.

\section{Results}

Demographic and clinical characteristics of ART-naïve youths at the China-Myanmar border

Overall, the pol gene was amplified successfully from 573 untreated youths with HIV-1; of these youths, 351 (61.26\%) were male, and $222(39.74 \%)$ were female. The most common infection route was sex among heterosexuals (70.51\%), followed by PWID (19.20\%) and MSM (8.9\%). The proportion of the MSM population is increasing annually ( 1 to $14 \%, p<0.001$ ). More than half of the youths were single $(58.81 \%)$, and the rest were married/cohabiting (37.35\%) or divorced (3.84\%). The subjects were Chinese (55.67\%) or Burmese (44.33\%). The proportion of PWID was higher among Burmese than among Chinese subjects $(29.13 \%$ vs. $11.29 \%, p<$ $0.001)$. The educational status of the subjects was mainly primary education $(28.27 \%)$ and junior high school education (27.23\%). The Chinese subjects had a higher rate of junior high school education (34.84\%) than the other subjects, and the Burmese subjects had a higher illiteracy rate $(38.58 \%)$ (Table 1$)$.

\section{High HIV genotype diversity among ART-naïve youths in China-Myanmar border areas}

The Stanford REGA online tool [21] was used to classify genotypes, and the classification was rechecked in the phylogenetic tree. There was a high distribution of URFs (44.08\%), including URF_BC (39.20\%), URF_01BC (3.48\%), and URF_01C (1.22\%), and 38.68\% CRF: including CRF01_AE (21.12\%), CRF07_BC (5.41\%), CRF08_BC (4.54\%), CRF5501B (1.39\%), CRF57BC (1.57\%), CRF62BC (1.05\%), CRF65cpx (2.09\%) and CRF64BC (1.57\%) (Fig. 1a), and $13.24 \%$ subtype $C$ and $4.04 \%$ subtype B. The genotypes changed over time: subtypes B and $C$ decreased from 2009 2017, while those of CRF01AE, CRF07BC and URFs continued to increase. According to the route of infection, the proportion of URF in PWID was higher than that observed in people who were infected with HIV-1 through sexual transmission (heterosexuals and MSM), but the proportions of CRF01AE and CRF08BC in cases of sexual transmission were high $(p<0.001)$. There are different HIV infection routes and genotypes between China and Myanmar, such as high URF in Burmese compared to Chinese $(34.48 \%$ vs. $56.08 \%, p<0.05)$ and high CRF07_BC and other CRFs in Chinese compared to Burmese (8.46\% vs. 1.57, $11.91 \%$ vs. $2.35 \% p<0.01$ ) (Fig. 1b).

\section{The HIV TDR among ART-naïve youths at the China-} Myanmar border increased significantly from 2009 to 2017 Thirty-six (36/573, 6.28\%) (2009 2011: 3.48\%, 2012 2013: 4.42\%, 2014 2015: 4.42\%, and 2016 2017: $9.48 \%)$ youths with TDRMs among the 573 untreated youths with HIV-1 were identified by the CPR program. 
Table 1 Clinical characterizes of untreated 16 25y youths infected HIV during 2009 2017

\begin{tabular}{|c|c|c|c|c|c|}
\hline & $\begin{array}{l}2009 \sim 2011 \\
n=115\end{array}$ & $\begin{array}{l}2012 \sim 2013 \\
n=113\end{array}$ & $\begin{array}{l}2014 \sim 2015 \\
n=113\end{array}$ & $\begin{array}{l}2016 \sim 2017 \\
n=232\end{array}$ & $\begin{array}{l}\text { Total } \\
N=573 \\
(100 \%)\end{array}$ \\
\hline \multicolumn{6}{|l|}{ Gender } \\
\hline Male & 53 & 54 & 71 & 173 & $351(61 \%)$ \\
\hline Female & 62 & 59 & 42 & 59 & $222(39 \%)$ \\
\hline \multicolumn{6}{|l|}{ Nationality } \\
\hline China & 90 & 64 & 47 & 118 & $319(56 \%)$ \\
\hline Myanmar & 25 & 49 & 66 & 114 & $254(44 \%)$ \\
\hline \multicolumn{6}{|l|}{ Route of transmission } \\
\hline Heterosexual & 88 & 80 & 78 & 158 & $404(71 \%)$ \\
\hline IDU & 19 & 26 & 25 & 40 & $110(19 \%)$ \\
\hline MSM & 1 & 7 & 9 & 34 & $51(9 \%)$ \\
\hline Unknown & 7 & 0 & 1 & 0 & $8(1 \%)$ \\
\hline \multicolumn{6}{|l|}{ Marriage status } \\
\hline Single & 51 & 53 & 72 & 161 & $337(59 \%)$ \\
\hline Married & 56 & 57 & 37 & 64 & $214(37 \%)$ \\
\hline Divorcelbereavement & 8 & 3 & 4 & 7 & $22(4 \%)$ \\
\hline \multicolumn{6}{|l|}{ Ethnicity } \\
\hline Han & 42 & 47 & 30 & 41 & $160(28 \%)$ \\
\hline Minorities & 73 & 66 & 83 & 191 & $413(72 \%)$ \\
\hline \multicolumn{6}{|l|}{ Education } \\
\hline Illiteracy & 24 & 29 & 22 & 40 & $115(20 \%)$ \\
\hline Primary school & 46 & 29 & 28 & 59 & $162(28 \%)$ \\
\hline Junior school & 38 & 39 & 38 & 41 & $156(27 \%)$ \\
\hline senior school & 4 & 2 & 7 & 20 & $33(6 \%)$ \\
\hline College and Above & 3 & 4 & 3 & 5 & $15(3 \%)$ \\
\hline Unknown & 0 & 10 & 15 & 67 & $92(16 \%)$ \\
\hline \multicolumn{6}{|l|}{ Job } \\
\hline Jobless & 11 & 12 & 14 & 21 & $58(10 \%)$ \\
\hline Farmer & 84 & 76 & 53 & 107 & $320(56 \%)$ \\
\hline Business services & 13 & 4 & 3 & 8 & $28(5 \%)$ \\
\hline Other & 7 & 9 & 27 & 25 & $68(12 \%)$ \\
\hline Unknown & 0 & 12 & 16 & 71 & $99(17 \%)$ \\
\hline
\end{tabular}

TDR increased significantly from 2009 to 2017 (3.48 to $9.48 \%, p<0.05$, Cochran-Armitage trend test). TDR among Chinese increased from 3.33 to $5.93 \%(p=0.37$, Cochran-Armitage trend test), and the highest percentage appeared in 2014 2015 (6.38\%). The TDR among Burmese increased significantly from 4.00 to $13.16 \%$ $(p<0.05$, Cochran-Armitage trend). TDR to NNRTIs (3.9 to $4.31 \%, p=0.57$ ) and PIs (0 to $0.86 \%, p=0.20)$ increased from 2009 to 2017. Moreover, the resistance to NRTIs increased significantly ( 0.87 to $5.17 \%, p=0.004$, Cochran-Armitage trend test) (Fig. 2a).

Overall, the average TDR to NNRTIs, NRTIs, and PIs was $3.49,2.62$, and $0.52 \%$, respectively. Most
(94.4\%, $n=34$ ) of HIV-1 patients with TDRM displayed a single drug class resistance mutation. Only two $(5.6 \%)$ strains harbored both NRTI and NNRTI mutations. No HIV-1 strain with TDRMs to triple classes of drugs was found in this study. Y181I/C and K103N were found in 7 and 9 infected individuals, respectively (Table 2). We used the Stanford HIVdb tool to assess the clinical impact of these mutations (excluding other polymorphism sites). Among NNRT Is, resistance to doravirine (DOR), etravirine (ETR), and rilpivirine (RPV) occurred mainly at a moderate level $(50,63$, and $78 \%$, respectively), and efavirenz (EFV) and nevirapine (NVP) had high-level resistance 


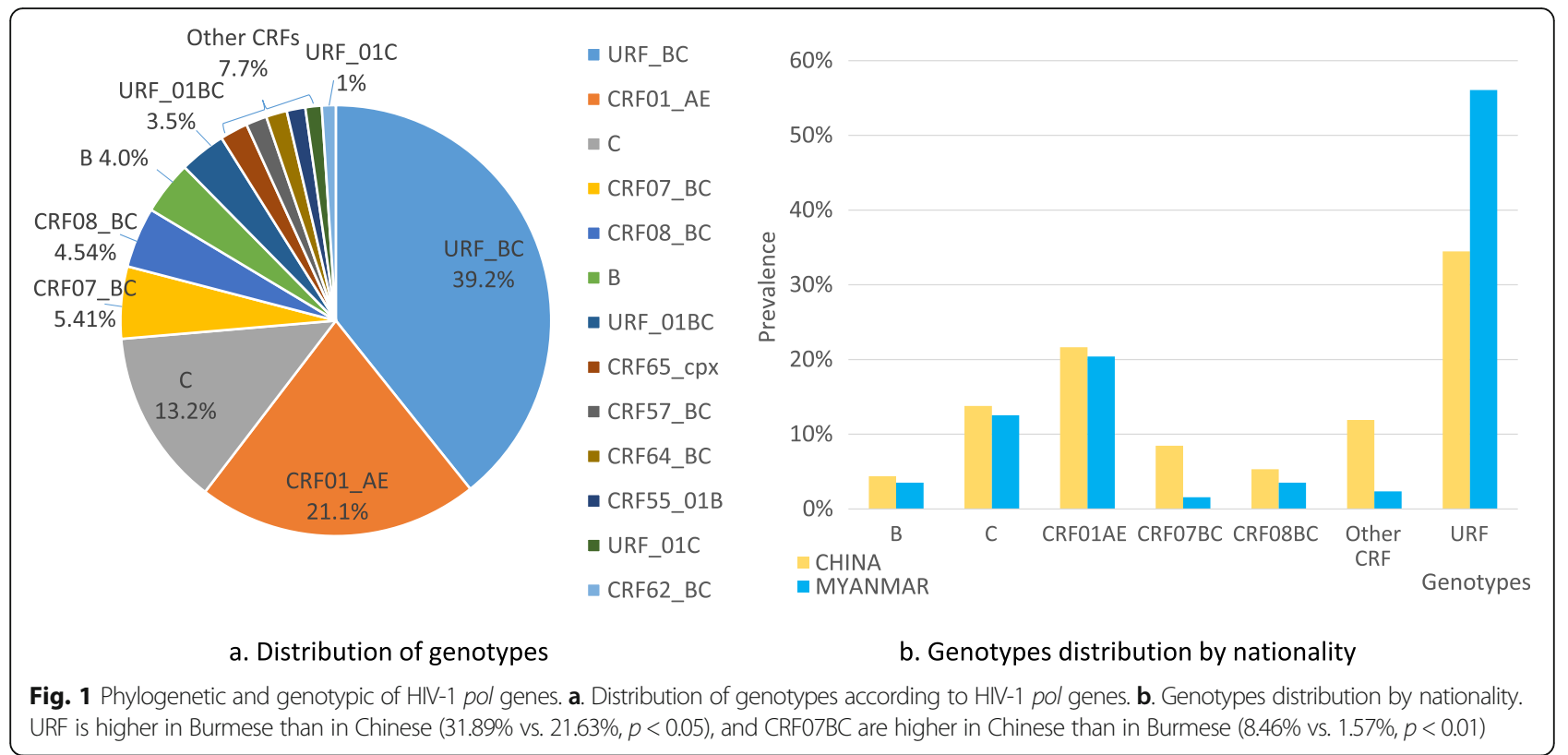

(55 and 95\%). Among NRTIs, except M184I (1), L74I (1), D67N (1), and T215I (1), the remaining mutations $(75 \%, 12 / 16)$ only conferred potential resistance (score $<15)$ to NRTIs. Only three youths carried TDRMs associated with PI resistance, and the I54M site caused broad resistance to PI drugs (Fig. 2b).

\section{Correlates of CD4 count and TDRMs among ART-naïve youths in the China-Myanmar border}

The CD4 counts of $461(80.45 \%)$ of the youths in the study were recorded. The average CD4 count was 483/ $\mathrm{mm}^{3}$ 95\% CI: $466.31 \sim 513.87 / \mathrm{mm}^{3}$. To examine the relationship between CD4 count and TDRMs, we divided

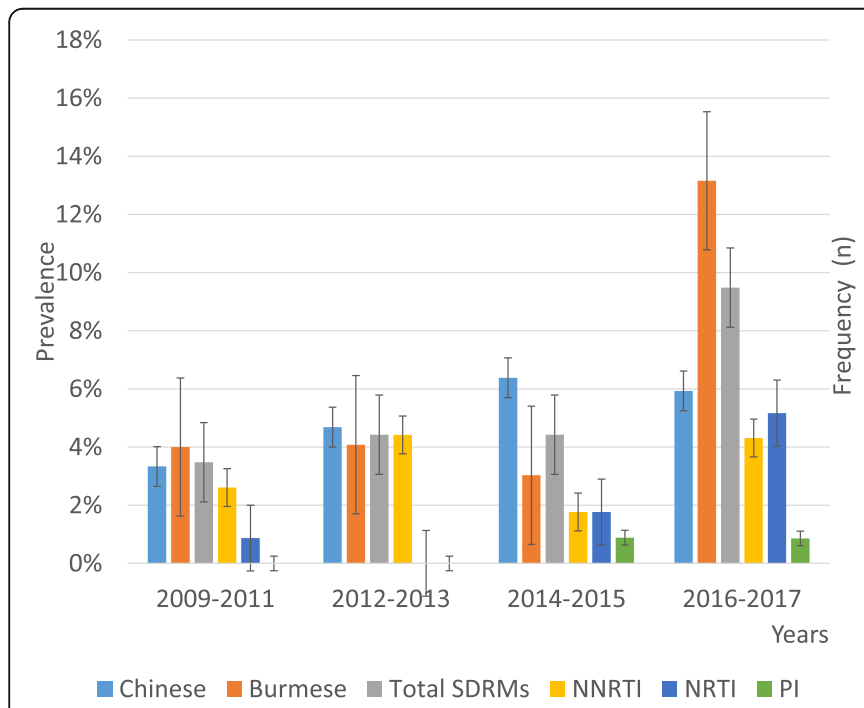

a. TDR $p$ revalence trend from 2009 2017

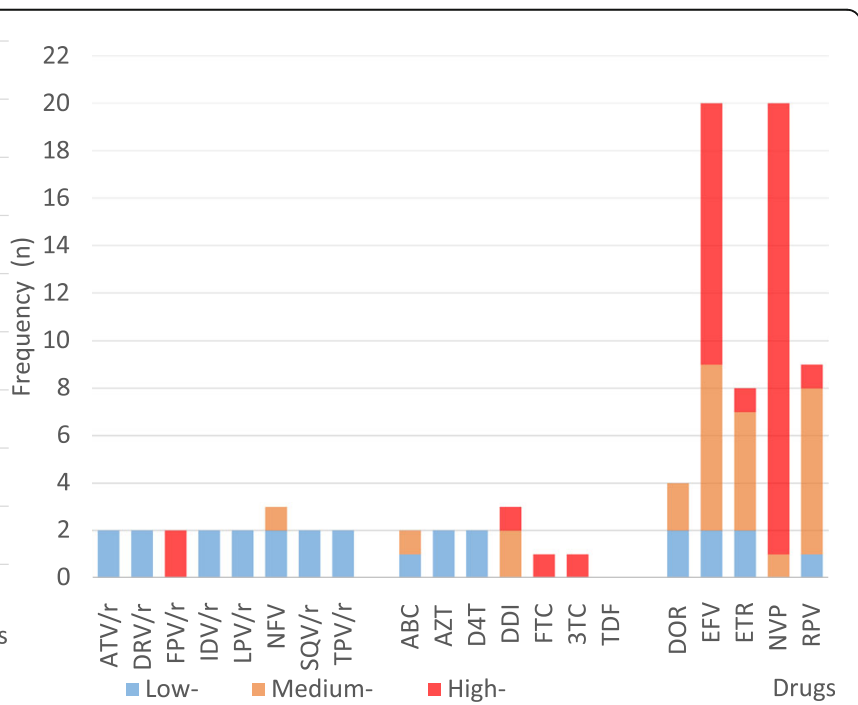

b. Resistance level of SDRMs

Fig. 2 Resistance level and TDR prevalence trend. a. TDR prevalence trend from $2009 \sim 2017$. Rates (mean, Std. Error, 95\% Cl) of TDR in Chinese: 2009-2011 (3/90, 3.30\%, Std. Error: 1.9, 0.00\% 7.60\%), 2012-2013 (3/64, 4.70\%, Std. Error: 2.66, 0.00\% 10.9\%), 2014-2015 (3/47, 6.4\%, Std. Error: 3.6, 0.00\% 14.6\%), 2016-2017 (7/118, 5.90\%, Std. Error: 2.20, 1.80\% 10.7\%); Burmese: 2009-2011 (1/25, 4.00\%, Std. Error: 4.00, 0.00\% 13.6\%), 2012-2013 (2/49, 4.10\%, Std. Error: 2.8, 0.00\% 10.4\%), 2014-2015 (2/66, 3.00\%, Std. Error: 2.1, 0.00\% 7.70\%), 2016-2017 (15/114, 13.20\%, Std. Error: $3.10,7.40 \% \sim 19.7 \%)$. b. Resistance levels of TDRMs. The degrees of resistance of the observed TDRMs to 20 ART drugs from the Stanford HIVDB program are distinguished by colors. The effects of other non-SDRMs in the sequence are excluded 
Table 2 Prevalence of HIV Transmitted Drug Resistance Mutations among untreated $16 \sim 25 y$ youths in Dehong during $2009 \sim 2017$

\begin{tabular}{ll}
\hline Type & DRM (\%) \\
\hline Total TDRMs & $38^{\mathrm{a}}$ \\
NRTI_TDRMs & $15(2.62 \%)$ \\
D67E & $4(0.70 \%)$ \\
D67N & $1(0.17 \%)$ \\
F77L & $3(0.52 \%)$ \\
K219N & $3(0.52 \%)$ \\
L74I & $1(0.17 \%)$ \\
M184I & $1(0.17 \%)$ \\
T215I & $1(0.17 \%)$ \\
T69D & $1(0.17 \%)$ \\
NNRTI_TDRMs & $20(3.49 \%)$ \\
G190A & $1(0.17 \%)$ \\
K101E & $1(0.17 \%)$ \\
K103N & $9(1.57 \%)$ \\
V106M & $2(0.35 \%)$ \\
Y181C & $7(1.22 \%)$ \\
PI_TDRMs & $3(0.52 \%)$ \\
I54M & $2(0.35 \%)$ \\
M46I & $1(0.17 \%)$ \\
\hline a & \\
\hline
\end{tabular}

a "Heterosexual" and "2016-2017" have two infectors who have two classes of TDRMs subjects into two groups: with or without TDRMs. The mean CD4 count was significantly lower in youths with TDRMs $\left(372.57 / \mathrm{mm}^{3}, 95 \%\right.$ CI: $\left.300.44 \sim 444.69 / \mathrm{mm}^{3}\right)$ than youths without TDRMs (496.08, 95\% CI: $471.91 \sim$ $520.25 / \mathrm{mm}^{3}$ ). K103N and $\mathrm{Y} 181 \mathrm{C}$ were the most common TDRMs in NNRTI. We divided subjects into the K103N group, Y181C group, NRTI group, PI group, and without TDRMs group. The K103N group, Y181C group, and NRTI_TDRMs group had lower mean CD4 counts $\left(337 / \mathrm{mm}^{3} 95 \%\right.$ CI: $246.42 \sim 427.57 / \mathrm{mm}^{3}, \quad 385 /$ $\mathrm{mm}^{3}$ 95\% CI: $132.54 \sim 637.85 / \mathrm{mm}^{3}$, and $363.6 / \mathrm{mm}^{3} 95 \%$ CI: $260.51 \sim 489.62 / \mathrm{mm}^{3}$, and $434 / \mathrm{mm}^{3} 95 \%$ CI: 130.69 $\sim 737.30 / \mathrm{mm}^{3}$, respectively) than the youths without TDRMs group (Table 3). None of the other characteristics or demographic information correlated with TRDMs (supplement Table S2).

\section{Discussion}

The city of Dehong is located in the China-Myanmar border area near the "Golden Triangle" and is a hotspot of HIV transmission and recombination, with a strong impact on the HIV-1 epidemic in China [12, 24]. From 2009 to 2017, 10,832 people were newly reported with HIV-1 infection at the Dehong border of China, 2210 $(20.40 \%)$ of whom were youths $(<25$ y). As youths are more likely to have recent infections and are highly representative of TDR, [16-18] we analyzed the TDR and genotype of untreated youths $(16 \sim 25$ y) newly diagnosed with HIV-1 infections over a 9-year period in Dehong.

The distribution of HIV-1 genotypes in China primarily includeCRF01AE, CRF07BC, CRF08BC, and B, while

Table 3 Correlates of CD4 count and DRMs among ART-naïve youths in the China-Myanmar border

\begin{tabular}{|c|c|c|c|c|c|c|}
\hline & \multirow[t]{2}{*}{$\mathrm{N}$} & \multirow[t]{2}{*}{ Mean $\left(\mathrm{mm}^{3}\right)$} & \multirow{2}{*}{$\begin{array}{l}\text { Standard Error } \\
\text { of Mean }\left(\mathrm{mm}^{3}\right)\end{array}$} & \multicolumn{2}{|l|}{$95 \% \mathrm{Cl}$ for Mean } & \multirow[t]{2}{*}{$P$} \\
\hline & & & & Lower Bound $\left(\mathrm{mm}^{3}\right)$ & Upper Bound $\left(\mathrm{mm}^{3}\right)$ & \\
\hline TDRMs & & & & & & 0.015 \\
\hline Without TDRMs & 431 & 496.08 & 12.13 & 471.91 & 520.25 & \\
\hline TDRMs & 30 & 372.57 & 34.05 & 300.44 & 444.69 & \\
\hline NNRTI_TDRMs & & & & & & 0.226 \\
\hline Without TDRMs & 431 & 496.08 & 12.13 & 471.91 & 520.25 & \\
\hline With K103N mutation & 9 & 337.00 & 39.05 & 246.42 & 427.57 & \\
\hline With Y181C mutation & 5 & 385.20 & 91.00 & 132.54 & 637.85 & \\
\hline Other mutation & 2 & 514.50 & NA & NA & NA & \\
\hline NRTI_TDRMs & & & & & & 0.064 \\
\hline Without TDRMs & 431 & 496.08 & 12.13 & 471.91 & 520.25 & \\
\hline NRTI_TDRMs & 13 & 363.60 & 54.39 & 260.51 & 489.62 & \\
\hline PI_TDRMs & & & & & & 0.674 \\
\hline Without TDRMs & 431 & 496.08 & 12.13 & 471.91 & 520.25 & \\
\hline PI_TDRMs & 3 & 434.00 & 82.70 & 130.69 & 737.30 & \\
\hline
\end{tabular}

The CD4 counts of 461 (80\%) of the youths in the study were recorded. The results of "Standard Error of Mean" and "95\% Cl for Mean" are based on 1000 bootstrap samples 
C, URF, and CRFs account for only a small proportion [9]. However, the distribution of genotypes differs in Dehong, which has a high prevalence of URFs [15, 25]. Similar to previous studies, the distribution of HIV genotypes in this study was diverse and complex. Notably, the prevalence of genotypes $\mathrm{B}$ and $\mathrm{C}$ decreased annually, and CRF01AE, CRF07BC, and URFs continues to increase. We also found that the proportion of URFs in Burmese and PWID populations was significantly higher than other populations. This prevalence result may indicate that due to the influence of drug injection in the "Golden Triangle", the presence of HIV-1 recombination networks occurred early among PWID in Dehong [26-28]. This has had a long-term impact on the HIV-1 epidemic in this area and made Dehong a hotspot for HIV recombination.

Previous studies indicated that frequent recombination was more effective than mutation in spreading drug resistance mutations $[29,30]$. Frequent communication around the China-Myanmar border has increased the frequency of recombination [15, 31]. However, this previous study was based on patients after treatment. Recombination allows the genome to combine beneficial mutations that existed before, which is conducive to the survival of viruses in the ARV. Our subjects were treatment-naïve, and there was no choice pressure of ARV drugs. This factor may be why the connection between TDR and reorganization was not significant $(p=0.793)$, despite the increasing reorganization. Overall, the average prevalence of TDR was $6.28 \%$, which exceeds the $5 \%$ moderate prevalence level. Since the early years of ART scale-up, TDR strains of HIV are likely to be limited, and all youths were ART-naive. The total number of TDRMs was small $(n=36)$, which may result in a statistical bias. We increased the sample capacity in 2016-2017 and observed a significant increase in TDR. Notably, the TDR was $9.48 \%$ in 2016-2017, which is significantly higher than the average TDR prevalence in China [3] and Myanmar [2]. The TDR in this study does not represent the average resistance level in China and Myanmar but it indicates the TDR increase among youths in hotspots of HIV transmission and recombination. In this study, found no significant difference in TDR prevalence between Burmese and Chinese subjects. The prevalence of TDR in Chinese subjects increased from 2009 to 2017 (from 3.92 to $5.93 \%$ ), the prevalence of TDR in Burmese migrants increased significantly from 2010 to 2017 (from 4.00 to 13.16\%). Burmese migrants are a key population for HIV prevention in this region.

Resistance to NNRTIs (2.92\%) was the most frequent TDRM. Among these mutations, K103N $(n=9)$ and Y181C/I $(n=7)$ were the most common TDRMs. These two mutations caused a high level of drug resistance to first-line treatment drugs (EFV and NVP). Among NRTI-related TDRMs (2.34\%), most $(75 \%, 12 / 16)$ exhibited only potential resistance. Azidothymidine (AZT), lamivudine (3TC), and tenofovir (TDF), as first-line NRTI drugs in China, have meager rates of transmission resistance $(0.30,0.15$, and $0 \%$, respectively). However, unlike the findings reported in other areas, [3, 32-37] NRTI resistance showed the most significant increase (from 0 to 5.17\%) from 2009 to 2017 in this study. Although the prevalence of TDR to PIs $(0.44 \%)$ was significantly lower than the prevalence of TDR to NNRTIs/ NRTIs, the I54M mutation caused universal resistance to PI drugs. These results suggest the need for considering resistance testing before initiating ART. We did not investigate integrase inhibitor (INSTI) TDR; these sequences were previously amplified and stored by our laboratory, and the primers did not include the INSTI region because INSTI drugs are not widely used in China and Myanmar. We will continue to increase the sample capacity and to monitor TDR (including INSTI) in the China-Myanmar border region.

Previous studies [38, 39] associated TDRMs with high CD4 counts, but our research found that HIV-1-infected youths with TDRMs had low CD4 counts. This discrepancy was also found in at least two other studies [40, 41]. A 2019 study [42] suggested that the detection of primary resistance was not associated with the speed of CD4 decline. We divided these six studies into two groups based on whether CD4 decreased. There were not many similarities in the resistance sites within the group. Notably, all of the studies had limitation. Infected persons at any stage of infection can enter the queue. The decline of $\mathrm{CD} 4$ was likely related to different stages of the disease course. In our study, the distribution of transmission routes and genotypes were similar to the overall HIV-1 situation in this region. Demographic information has no correlation with TRDMs, which minimized the error caused by sampling. Moreover, our survey objects were newly reported ART-naïve youths, which reduces the error of infection time as possible and excludes individuals with long-standing infections or prior ART. Therefore, this relationship between CD4 count and TDRMs may be generalized to individuals infected with HIV-1 at the China-Myanmar border. We compared the CD4 count between NNRTI/NRTI/PI mutation and nonTDRM youths. Although all groups with TDRMs showed a lower mean CD4 count than the group without TDRMs, the difference was not statistically significant. Due to the small number of other TDRMs, we could not determine the correlation between other single TDRMs and CD4 counts. In summary, large sample size and more epidemiological data are required to evaluate the potential role of three classes of TDR (NNRTI/NRTI/PI) or K103N, Y181C in affecting the decline of $\mathrm{CD} 4$ count.

\section{Conclusion}

TDR increased two-fold and exceeded the 5\% moderate prevalence level from 2009 to 2017 among untreated 
youths in the China-Myanmar border, primarily resistance to NNRTIs and NRTIs. HIV-1 genotypes are diverse and complex, with URFs, CRF01AE as the predominant genotypes. The HIV-1 TDR increase and a low CD4 count of patients with TDRMs suggest the need for considering drug resistance before initiating ART in HIV recombination hotspots.

\section{Supplementary Information}

The online version contains supplementary material available at https://doi. org/10.1186/s12879-021-05794-5.

Additional file 1: Table S1. Age distribution of newly reported HIV infections in Dehong city, during $2009 \sim 2017$. Table S2. Demography and clinical characteristics of untreated $16 \sim 25 y$ youths infected HIV with or without TDRMs.

\begin{abstract}
Abbreviations
HIV: Human immunodeficiency virus; TDR: Transmitted drug resistance; ADR: Acquired drug resistance; PLWH: People living with HIV; WHO: World Health Organization; ART: Antiretroviral therapy; NNRTI: Non-nucleoside reverse-transcriptase inhibitor; NRTI: Nucleoside reverse-transcriptase inhibitor; PI: Protease inhibitor; PWID: People who injected drug; PrEP: Pre-exposure prophylaxis; EFV: Efavirenz; NVP: Nevirapine; FTC: Emtricitabine; 3TC: Lamivudine; ABC: Abacavir; DDI: Didanosine; D4T: Stavudine; TDF: Tenofovir; AZT: Azidothymidine; DRV/r: Darunavir; LPV/r: Lopinavir; ATV/ r: Atazanavir; DRM: Drug resistance mutation; HIVDR: HIV Drug Resistance Database; 95\% Cl: 95\% Confidence interval
\end{abstract}

\section{Acknowledgements}

Not applicable.

\section{Authors' contributions}

L.M. M.H. conceived and designed the experiments; M.C., J.W., Y.Y., Y.M. collected samples. Y.D. M.C. Y.Z., L.W., Q.L. performed the experiments; Y.D., Y.F., H.X., S.D. analyzed the data; Y.D., L.M. M.H. wrote the paper; all authors discussed the results and contributed to the final manuscript. The author(s) read and approved the final manuscript.

\section{Funding}

This work was supported by the National Science and Technology Major Project (Grant 2018ZX10101002-004-003), the National Natural Science Foundation of China (Grants 81871694, 81773447, 81561128006), and the National Major Project of the State Key Laboratory of Infectious Diseases Prevention and Control (Grant 2011SKLID102). The funding body had no role in the data collection, analysis, and interpretation of the verbal data, and writing of the manuscript.

\section{Availability of data and materials}

The datasets used during the current study are available from the corresponding author on reasonable request.

\section{Ethics approval and consent to participate}

The ethical review of this study was formally approved by and obtained written consent (approval no. X190111549) from the Medical Ethics Certification Committee of the Chinese Center for Disease Control and Prevention. Written informed consent was obtained from all subjects included in the study.

\section{Consent for publication}

Not applicable.

\section{Competing interests}

The authors report no conflicts of interest in this work.

\section{Author details}

${ }^{1}$ State Key Laboratory of Infectious Disease Prevention and Control, National Center for AIDS/STD Control and Prevention, Collaborative Innovation Center for Diagnosis and Treatment of Infectious Diseases, Chinese Center for Disease Control and Prevention, 155 Changbai Road, Changping District, Beijing 102206, China. ${ }^{2}$ Institute for AIDS/STD Control and Prevention, Yunnan Center for Disease Control and Prevention, No. 158, Dongsi Street, Xishan District, Kunming 650022, Yunnan Province, China. ${ }^{3}$ Dehong Dai and Jingpo Autonomous Prefecture Center for Disease Control and Prevention, Mangshi 678400, China.

Received: 19 September 2020 Accepted: 12 January 2021

Published online: 21 January 2021

\section{References}

1. Latest global and regional statistics on the status of the AIDS epidemic. [https://www.unaids.org/en/resources/documents/2019/UNAIDS_FactSheet].

2. HIV drug resistance report 2019 [https:/apps.who.int/iris/handle/10665/325891].

3. Ye J, Hao M, Xing H, Zhang F, Wu H, Lv W, Jiang T, Wang Y, Wang J, Feng $Y$, et al. Transmitted HIV drug resistance among individuals with newly diagnosed HIV infection: a multicenter observational study. AIDS. 2019.

4. Yang LIJZ, Yi FENG, Hongyan LU. Zang xihui, Xu xiaoqin, Yang Jiezhe: gene characteristic and transmitted drug resistance of HIV-1 strains among infected patientsat the age of 16-25 years in China. China J AIDS STD. 2017; 23(03):176-80

5. Chen $M$, Zhu $Q$, Xing $H$, Chen $H$, Jin X, Dong L, Dai J, Yang M, Yang C, Jia $M$, et al. The characteristics of pretreatment HIV-1 drug resistance in western Yunnan, China. Epidemiol Infect. 2020;148:e102.

6. Chen M, Ma Y, Chen H, Dai J, Dong L, Yang C, Li Y, Luo H, Zhang R, Jin X, et al. HIV-1 genetic transmission networks among men who have sex with men in Kunming, China. PLoS One. 2018;13(4):e0196548.

7. World Drug Report 2016, united nations office on drugs and crime. [http:// www.unodc.org/wdr2016/].

8. Ma Y, Li Z, Zhang K, Yang W, X R: HIV was first discovered among injection drug users in China. Chin J Epidemiology 1990, 11: 184-185.

9. Li X, Li W, Zhong P, Fang K, Zhu K, Musa TH, Song Y, Du G, Gao R, Guo Y, et al. Nationwide trends in molecular epidemiology of HIV-1 in China. AIDS Res Hum Retrovir. 2016;32(9):851-9.

10. Jia Y, Sun J, Fan L, Song D, Tian S, Yang Y, Jia M, Lu L, Sun X, Zhang S, et al. Estimates of HIV prevalence in a highly endemic area of China: Dehong prefecture, Yunnan Province. Int J Epidemiol. 2008;37(6):1287-96.

11. Luo CC, Tian C, Hu DJ, Kai M, Dondero T, Zheng X. HIV-1 subtype C in China. Lancet. 1995;345(8956):1051-2.

12. Feng $Y$, He X, Hsi JH, Li F, Li X, Wang Q, Ruan Y, Xing H, Lam T, Pybus OG, et al. The rapidly expanding CRF01_AE epidemic in China is driven by multiple lineages of HIV-1 viruses introduced in the 1990s. AIDS. 2013; 27(11):1793-802.

13. Tee KK, Pybus OG, Li XJ, Han X, Shang H, Kamarulzaman A, Takebe Y. Temporal and spatial dynamics of human immunodeficiency virus type 1 circulating recombinant forms 08_BC and 07_BC in Asia. J Virol. 2008;82(18): 9206-15.

14. Li Z, He X, Wang Z, Xing H, Li F, Yang Y, Wang Q, Takebe Y, Shao Y. Tracing the origin and history of HIV-1 subtype B' epidemic by near full-length genome analyses. AIDS. 2012;26(7):877-84.

15. Wei H, Xing H, Hsi JH, Jia M, Feng Y, Duan S, He C, Yao S, Ruan Y, He X, et al. The sexually driven epidemic in youths in China's southwestern border region was caused by dynamic emerging multiple recombinant HIV1 strains. Sci Rep. 2015;5:11323.

16. World Health O. World Health Organization protocol for surveillance of transmitted HIV drug resistance: 2012 update. In. Geneva: World Health Organization; 2012.

17. Bennett DE, Myatt M, Bertagnolio S, Sutherland D, Gilks CF. Recommendations for surveillance of transmitted HIV drug resistance in countries scaling up antiretroviral treatment. Antivir Ther (Lond). 2008; 13(Suppl 2):25-36.

18. HIV drug resistance surveillance guidance [https://apps.who.int/iris/handle/1 0665/204471]

19. Zhou Z, Wagar N, DeVos JR, Rottinghaus E, Diallo K, Nguyen DB, Bassey O, Ugbena R, Wadonda-Kabondo N, McConnell MS, et al. Optimization of a low cost and broadly sensitive genotyping assay for HIV-1 drug resistance 
surveillance and monitoring in resource-limited settings. PLoS One. 2011; 6(11):e28184.

20. Stamatakis A. RAxML version 8: a tool for phylogenetic analysis and postanalysis of large phylogenies. Bioinformatics. 2014;30(9):1312-3.

21. Pineda-Pena AC, Faria NR, Imbrechts S, Libin P, Abecasis AB, Deforche $K$, Gomez-Lopez A, Camacho RJ, de Oliveira T, Vandamme AM. Automated subtyping of HIV-1 genetic sequences for clinical and surveillance purposes: performance evaluation of the new REGA version 3 and seven other tools. Infect Genet Evol. 2013;19:337-48.

22. Gifford RJ, Liu TF, Rhee SY, Kiuchi M, Hue S, Pillay D, Shafer RW. The calibrated population resistance tool: standardized genotypic estimation of transmitted HIV-1 drug resistance. Bioinformatics. 2009;25(9):1197-8.

23. Liu TF, Shafer RW. Web resources for HIV type 1 genotypic-resistance test interpretation. Clin Infect Dis. 2006;42(11):1608-18.

24. Feng $Y$, Takebe $Y$, Wei H, He X, Hsi JH, Li Z, Xing H, Ruan Y, Yang Y, Li F, et al. Geographic origin and evolutionary history of China's two predominant HIV-1 circulating recombinant forms, CRF07_BC and CRF08_ BC. Sci Rep. 2016;6:19279.

25. Chen M, Ma Y, Duan S, Xing H, Yao S, Su Y, Luo H, Yang L, Chen H, Fu L, et al. Genetic diversity and drug resistance among newly diagnosed and antiretroviral treatment-naive HIV-infected individuals in western Yunnan: a hot area of viral recombination in China. BMC Infect Dis. 2012;12:382.

26. Yang $R$, Xia X, Kusagawa S, Zhang C, Ben K, Takebe Y. On-going generation of multiple forms of HIV-1 intersubtype recombinants in the Yunnan Province of China. AIDS. 2002;16(10):1401-7.

27. Bar KJ, Li H, Chamberland A, Tremblay C, Routy JP, Grayson T, Sun C, Wang $\mathrm{S}$, Learn GH, Morgan CJ, et al. Wide variation in the multiplicity of HIV-1 infection among injection drug users. J Virol. 2010;84(12):6241-7.

28. Delviks-Frankenberry K, Galli A, Nikolaitchik O, Mens H, Pathak VK, Hu WS. Mechanisms and factors that influence high frequency retroviral recombination. Viruses. 2011;3(9):1650-80.

29. Carvajal-Rodriguez A, Crandall KA, Posada D. Recombination favors the evolution of drug resistance in HIV-1 during antiretroviral therapy. Infect Genet Evol. 2007;7(4):476-83.

30. Rouzine IM, Coffin JM. Evolution of human immunodeficiency virus under selection and weak recombination. Genetics. 2005;170(1):7-18.

31. Liu J, Jia Y, Xu Q, Zheng YT, Zhang C. Phylodynamics of HIV-1 unique recombinant forms in China-Myanmar border: implication for HIV-1 transmission to Myanmar from Dehong, China. Infect Genet Evol. 2012;12(8): 1944-8.

32. Park M, Kee MK, Rhee J, Park JY, Kim SS, Kang C, Choi JY. The trend of transmitted drug resistance in newly diagnosed antiretroviral-naive HIV/ AIDS patients during 1999-2012 in South Korea. J Clin Virol. 2016;81:53-7.

33. Trebelcock WL, Lama JR, Duerr A, Sanchez H, Cabello R, Gilada T, Segura P, Reisner SL, Mayer KH, Mullins J, et al. HIV pretreatment drug resistance among cisgender MSM and transgender women from Lima, Peru. J Int AIDS Soc. 2019;22(11):e25411.

34. Andersson E, Nordquist A, Esbjornsson J, Flamholc L, Gisslen M, Hejdeman B, Marrone G, Norrgren H, Svedhem V, Wendahl S, et al. Increase in transmitted drug resistance in migrants from sub-Saharan Africa diagnosed with HIV-1 in Sweden. AIDS. 2018;32(7):877-84

35. Rocheleau G, Brumme CJ, Shoveller J, Lima VD, Harrigan PR. Longitudinal trends of HIV drug resistance in a large Canadian cohort, 1996-2016. Clin Microbiol Infect. 2018;24(2):185-91.

36. D'Costa J, Gooey M, Richards N, Sameer R, Lee E, Chibo D. Analysis of transmitted HIV drug resistance from 2005 to 2015 in Victoria, Australia: a comparison of the old and the new. Sex Health. 2017;14(6):558-65.

37. Ferreira ACG, Coelho LE, Grinsztejn E, Jesus CS, Guimaraes ML, Veloso VG, Grinsztejn B, Cardoso SW. Transmitted drug resistance in patients with acute/recent HIV infection in Brazil. Braz J Infect Dis. 2017;21(4):396-401.

38. Grant RM, Hecht FM, Warmerdam M, Liu L, Liegler T, Petropoulos CJ, Hellmann NS, Chesney M, Busch MP, Kahn JO. Time trends in primary HIV-1 drug resistance among recently infected persons. JAMA. 2002;288(2):181-8.

39. Booth CL, Garcia-Diaz AM, Youle MS, Johnson MA, Phillips A, Geretti AM. Prevalence and predictors of antiretroviral drug resistance in newly diagnosed HIV-1 infection. J Antimicrob Chemother. 2007;59(3):517-24.

40. Panichsillapakit T, Smith DM, Wertheim JO, Richman DD, Little SJ, Mehta SR. Prevalence of transmitted HIV drug resistance among recently infected persons in San Diego, CA 1996-2013. J Acquir Immune Defic Syndr. 2016; 71(2):228-36.
41. Sungkanuparph S, Oyomopito R, Sirivichayakul S, Sirisanthana T, Li PC, Kantipong P, Lee CK, Kamarulzaman A, Messerschmidt L, Law MG, et al. HIV1 drug resistance mutations among antiretroviral-naive HIV-1-infected patients in Asia: results from the TREAT Asia studies to evaluate resistancemonitoring study. Clin Infect Dis. 2011;52(8):1053-7.

42. Schultze A, Torti C, Cozzi-Lepri A, Vandamme AM, Zazzi M, Sambatakou H, De Luca A, Geretti AM, Sonnerborg A, Ruiz L, et al. The effect of primary drug resistance on CD4+ cell decline and the viral load set-point in HIV positive individuals before the start of antiretroviral therapy. AIDS. 2019; 33(2):315-26.

\section{Publisher's Note}

Springer Nature remains neutral with regard to jurisdictional claims in published maps and institutional affiliations.
Ready to submit your research? Choose BMC and benefit from:

- fast, convenient online submission

- thorough peer review by experienced researchers in your field

- rapid publication on acceptance

- support for research data, including large and complex data types

- gold Open Access which fosters wider collaboration and increased citations

- maximum visibility for your research: over $100 \mathrm{M}$ website views per year

At BMC, research is always in progress.

Learn more biomedcentral.com/submissions 